In: "Recent Advances in Computational Sciences," P.E.T. Jorgensen, X. Shen, C.-W. Shu, and N. Yan, eds., World Scientific, Singapore (2008), pp. 209-217.

\title{
CONVOLUTION AND WIENER AMALGAM SPACES ON THE AFFINE GROUP
}

\author{
CHRISTOPHER HEIL* \\ School of Mathematics, \\ Georgia Institute of Technology, \\ Atlanta, Georgia 30332-0160 USA \\ E-mail: heil@math.gatech.edu \\ GITTA KUTYNIOK ${ }^{\dagger}$ \\ Institute of Mathematics, \\ Justus-Liebig-University Giessen, \\ 35392 Giessen, Germany \\ E-mail: gitta.kutyniok@math.uni-giessen.de
}

\begin{abstract}
Wiener amalgam spaces are a class of spaces of functions or distributions defined by a norm which amalgamates a local criterion for membership in the space with a global criterion. This article presents a proof of a useful convolution relation for amalgam spaces on the affine group.
\end{abstract}

\section{Introduction}

Wiener amalgam spaces are a class of spaces of functions or distributions defined by a norm which amalgamates a local criterion for membership in the space with a global criterion. Versions of such amalgam spaces have arisen independently many times in the literature, and often provide a natural and compelling context for formulating results. As one illustration of the shortcomings of the usual Lebesgue space $L^{p}(\mathbb{R})$ in regard to distinguishing between local and global properties of functions, note that all rearrangements of a function have identical $L^{p}$ norms. Hence it is not possible to recognize from its norm whether a function is the characteristic

*Partially supported by NSF grant DMS-0139261.

${ }^{\dagger}$ Partially supported by DFG research fellowship KU 1446/5. 
function of an interval or the sum of many characteristic functions of small intervals spread widely over $\mathbb{R}$.

The first amalgam spaces were introduced by Wiener in his study of generalized harmonic analysis. In particular, Wiener defined in Ref. 6 the amalgams on the real line that we now call $W\left(L^{1}, L^{2}\right)$ and $W\left(L^{2}, L^{1}\right)$, and in Refs. 7,8 he defined the spaces $W\left(L^{1}, L^{\infty}\right)$ and $W\left(L^{\infty}, L^{1}\right)$, using what we will refer to as a discrete norm for these spaces, namely,

$$
\|f\|_{W\left(L^{p}, L^{q}\right)}=\left(\sum_{n \in \mathbb{Z}}\left(\int_{n}^{n+1}|f(t)|^{p} d t\right)^{q / p}\right)^{1 / q},
$$

with the usual adjustments if $p$ or $q$ is infinity.

A comprehensive general theory of amalgam spaces $W(B, C)$ on a locally compact group $G$ was introduced and extensively studied by Feichtinger, see Refs. $1,2,3$. Here $B$ is a Banach function space on $G$ whose norm corresponds to the "local" component of the amalgam, and the Banach function space $C$ corresponds to the global component. For an expository introduction to Wiener amalgams on $\mathbb{R}$ with extensive references to the original literature, we refer to Ref. 4.

Wiener amalgam spaces have a number of useful properties. In this article we focus on convolution relations. Let $G$ be a locally compact group. We say that $G$ is an IN-group ("IN" for "invariant neighborhoods") if there exists a neighborhood $Q$ of the identity with compact closure that is invariant under all inner automorphisms. That is, we must have $x Q x^{-1}=Q$ for all $x \in G$. In this case, if $B_{1} * B_{2} \subseteq B_{3}$ and $C_{1} * C_{2} \subseteq C_{3}$, then we have that

$$
W\left(B_{1}, C_{1}\right) * W\left(B_{2}, C_{2}\right) \subseteq W\left(B_{3}, C_{3}\right) .
$$

The IN-groups include all abelian groups, and some non-abelian groups such as the reduced Heisenberg group (which is important for time-frequency analysis). Unfortunately, the affine or $a x+b$ group (which is important for wavelet theory) is not an IN-group.

However, there are still interesting convolution relations that do hold for amalgam spaces on non-IN groups. In particular, one such convolution result played an important role in our recent work in Ref. 5 on density conditions and the Homogenous Approximation Property for wavelet frames, namely,

$$
L^{1} * W\left(L^{\infty}, L^{1}\right) \subseteq W\left(L^{\infty}, L^{1}\right)
$$


This particular convolution result was proved by Feichtinger and Gröchenig in Ref. 2 for general locally compact groups. It is our purpose in this expository article to give a clear and mostly self-contained proof of this useful convolution relation for the particular case of the affine group. We emphasize that this result is due to Feichtinger and Gröchenig, not to ourselves, and the proof given here elaborates on the proof given in Ref. 2 .

This paper is organized as follows. In Section 2 we present some background on the affine group and amalgam spaces on the affine group. In Section 3 we prove the convolution relation for the affine group.

\section{Notation and Preliminary Results}

\subsection{General Notation}

Let $\mathbb{A}=\mathbb{R}^{+} \times \mathbb{R}$ denote the affine group, endowed with the multiplication

$$
(a, b)(x, y)=\left(a x, \frac{b}{x}+y\right) .
$$

The identity element of $\mathbb{A}$ is $e=(1,0)$, and inverses are given by

$$
(a, b)^{-1}=\left(\frac{1}{a},-a b\right) .
$$

The left-invariant Haar measure on $\mathbb{A}$ is $\mu=\frac{d x}{x} d y$. We denote the norm on $L^{p}(\mathbb{A})$ with respect to this Haar measure by $\|\cdot\|_{L^{p}(\mathbb{A})}$, whereas the norm on $L^{p}(\mathbb{R})$ will be denoted by $\|\cdot\|_{p}$.

We define left-translation, right-translation, and a renormalized righttranslation on the affine group by

$$
\begin{aligned}
& L_{(a, b)} F(x, y)=F\left((a, b)^{-1}(x, y)\right), \\
& R_{(a, b)} F(x, y)=F\left((x, y)(a, b)^{-1}\right), \\
& A_{(a, b)} F(x, y)=a R_{(a, b)} F(x, y)=a F\left((x, y)(a, b)^{-1}\right) .
\end{aligned}
$$

The (left) convolution of two functions $F$ and $G$ on the affine group is defined by

$$
\begin{aligned}
(F * G)(x, y) & =\iint_{\mathbb{A}} F(a, b) L_{(a, b)} G(x, y) \frac{d a}{a} d b \\
& =\iint_{\mathbb{A}} F(a, b) G\left((a, b)^{-1}(x, y)\right) \frac{d a}{a} d b,
\end{aligned}
$$

whenever this exists. 


\subsection{Preliminary Lemmas}

We will need the following lemma on the properties of the left Haar measure on the affine group with respect to left-translations, right-translations, and inverses.

Lemma 2.1. Let $F \in L^{1}(\mathbb{A})$ be given.
(a) $\iint_{\mathbb{A}} F((a, b)(x, y)) \frac{d x}{x} d y=\iint_{\mathbb{A}} F(x, y) \frac{d x}{x} d y$.
(b) $\iint_{\mathbb{A}} F((x, y)(a, b)) \frac{d x}{x} d y=a \iint_{\mathbb{A}} F(x, y) \frac{d x}{x} d y$.
(c) $\iint_{\mathbb{A}} F\left((x, y)^{-1}\right) \frac{d x}{x} d y=\iint_{\mathbb{A}} x F(x, y) \frac{d x}{x} d y$.
(d) $\left\|A_{(a, b)} F\right\|_{L^{1}(\mathbb{A})}=\|F\|_{L^{1}(\mathbb{A})}$.

Proof. These follow by making appropriate changes of variable in the integrals. We illustrate by proving parts (b) and (d).

(b) We have

$$
\begin{aligned}
\iint_{\mathbb{A}} F((x, y)(a, b)) \frac{d x}{x} d y & =\int_{-\infty}^{\infty} \int_{0}^{\infty} F\left(x a, \frac{y}{a}+b\right) \frac{d x}{x} d y \\
& =\int_{-\infty}^{\infty} \int_{0}^{\infty} F\left(p, \frac{y}{a}+b\right) \frac{d p}{p} d y \quad(\operatorname{set} p=x a) \\
& =\int_{0}^{\infty} \int_{-\infty}^{\infty} F\left(p, \frac{y}{a}+b\right) d y \frac{d p}{p} \\
& =\int_{-\infty}^{\infty} \int_{0}^{\infty} F(p, q) a d q \frac{d p}{p} \quad\left(\operatorname{set} q=\frac{y}{a}+b\right) \\
& =a \int_{-\infty}^{\infty} \int_{0}^{\infty} F(p, q) \frac{d p}{p} d q .
\end{aligned}
$$

(d) We have

$$
\begin{aligned}
\left\|A_{(a, b)} F\right\|_{L^{1}(\mathbb{A})} & =\iint_{\mathbb{A}}\left|A_{(a, b)} F(x, y)\right| \frac{d x}{x} d y \\
& =\iint_{\mathbb{A}} a\left|F\left((x, y)(a, b)^{-1}\right)\right| \frac{d x}{x} d y \\
& =\iint_{\mathbb{A}} a\left|F\left((x, y)\left(\frac{1}{a},-a b\right)\right)\right| \frac{d x}{x} d y
\end{aligned}
$$




$$
\begin{aligned}
& =\iint_{\mathbb{A}} a|F(x, y)| \frac{1}{a} \frac{d x}{x} d y \quad \text { (by part (b)) } \\
& =\|F\|_{L^{1}(\mathbb{A})} .
\end{aligned}
$$

\subsection{Lemmas on Convolution}

We need the following lemmas regarding convolution on the affine group.

Lemma 2.2. If $F, G \in L^{1}(\mathbb{A})$ then $F * G \in L^{1}(\mathbb{A})$, and

$$
\|F * G\|_{L^{1}(\mathbb{A})} \leq\|F\|_{L^{1}(\mathbb{A})}\|G\|_{L^{1}(\mathbb{A})} .
$$

\section{Proof.}

$$
\begin{aligned}
\|F * G\|_{L^{1}(\mathbb{A})} & =\iint_{\mathbb{A}}|F * G(x, y)| \frac{d x}{x} d y \\
& \leq \iint_{\mathbb{A}} \iint_{\mathbb{A}}|F(a, b)|\left|G\left((a, b)^{-1}(x, y)\right)\right| \frac{d x}{x} d y \frac{d a}{a} d b \\
& =\iint_{\mathbb{A}}|F(a, b)| \iint_{\mathbb{A}}|G(x, y)| \frac{d x}{x} d y \frac{d a}{a} d b \\
& =\|F\|_{L^{1}(\mathbb{A})}\|G\|_{L^{1}(\mathbb{A})} .
\end{aligned}
$$

Lemma 2.3. If $F, G \in L^{1}(\mathbb{A})$, then

$$
A_{(a, b)} F * G=F * L_{(a, b)} G .
$$

Proof.

$$
\begin{aligned}
\left(A_{(a, b)} F * G\right)(x, y) & =\iint_{\mathbb{A}} A_{(a, b)} F(p, q) G\left((p, q)^{-1}(x, y)\right) \frac{d p}{p} d q \\
& =\iint_{\mathbb{A}} a F\left((p, q)(a, b)^{-1}\right) G\left((p, q)^{-1}(x, y)\right) \frac{d p}{p} d q \\
& =\iint_{\mathbb{A}} F(p, q) G\left([(p, q)(a, b)]^{-1}(x, y)\right) \frac{d p}{p} d q \\
& =\iint_{\mathbb{A}} F(p, q) G\left((a, b)^{-1}(p, q)^{-1}(x, y)\right) \frac{d p}{p} d q \\
& =\iint_{\mathbb{A}} F(p, q)\left(L_{(a, b)} G\right)\left((p, q)^{-1}(x, y)\right) \frac{d p}{p} d q \\
& =\left(F * L_{(a, b)} G\right)(x, y) .
\end{aligned}
$$




\subsection{Amalgam Spaces on the Affine Group}

For our purposes, we will need the following particular amalgam spaces on the affine group.

Definition 2.1. Given $1 \leq p<\infty$, the amalgam space $W_{\mathbb{A}}\left(L^{\infty}, L^{p}\right)$ on the affine group consists of all functions $F: \mathbb{A} \rightarrow \mathbb{C}$ such that the norm

$$
\|F\|_{W_{\mathbb{A}}\left(L^{\infty}, L^{p}\right)}=\left(\iint_{\mathbb{A}} \operatorname{esssup}_{(a, b) \in \mathbb{A}}\left|F(a, b) \Phi\left((x, y)^{-1}(a, b)\right)\right|^{p} \frac{d x}{x} d y\right)^{1 / p}
$$

is finite, where $\Phi$ is a fixed continuous function with compact support satisfying $0 \leq \Phi(x, y) \leq 1$ for all $(x, y) \in \mathbb{A}$ and $\Phi(x, y)=1$ on some compact neighborhood of the identity. The amalgam space $W_{\mathbb{A}}\left(\mathcal{C}, L^{p}\right)$ is the closed subspace of $W_{\mathbb{A}}\left(L^{\infty}, L^{p}\right)$ consisting of the continuous functions in $W_{\mathbb{A}}\left(L^{\infty}, L^{p}\right)$.

$W_{\mathbb{A}}\left(L^{\infty}, L^{p}\right)$ is a Banach space, and its definition is independent of the choice of $\Phi$, in the sense that each choice of $\Phi$ yields the same space under an equivalent norm. For proofs and more details, see Refs. 1, 2.

We need the following fact regarding equivalent discrete-type norms for the amalgam spaces. The proof is similar to the construction of the sets $B_{j k}$ in Ref. 5.

Proposition 2.1. There exists a compact neighborhood $Q$ of $(1,0)$ in $\mathbb{A}$ with $Q=Q^{-1}$ and there exist points $\left(p_{n}, q_{n}\right) \in \mathbb{A}, n \in \mathbb{N}$, such that the following hold.

(a) If $G \in W_{\mathbb{A}}\left(L^{\infty}, L^{1}\right)$ then there exist functions $G_{n} \in L^{\infty}(\mathbb{A})$ with $\operatorname{supp}\left(G_{n}\right) \subseteq Q$ such that $G=\sum_{n} L_{\left(p_{n}, q_{n}\right)} G_{n}$.

(b) The following is an equivalent norm on $W_{\mathbb{A}}\left(L^{\infty}, L^{1}\right)$ :

$$
\|G\|_{W_{\mathbb{A}}\left(L^{\infty}, L^{1}\right)}=\sum_{n \in \mathbb{N}}\left\|G_{n}\right\|_{L^{\infty}(\mathbb{A})} .
$$

\section{Amalgam Spaces and Convolution on the Affine Group}

Now we can prove our main result.

Theorem 3.1. $L^{1}(\mathbb{A}) * W_{\mathbb{A}}\left(L^{\infty}, L^{1}\right) \subseteq W_{\mathbb{A}}\left(L^{\infty}, L^{1}\right)$.

Proof. Let $\Phi$ be any fixed function with compact support in $\mathbb{A}$ satisfying $0 \leq \Phi(x, y) \leq 1$ for all $(x, y) \in \mathbb{A}$ and $\Phi(x, y)=1$ on some compact neighborhood of the identity, 
Let $F \in L^{1}(\mathbb{A})$ and $G \in W_{\mathbb{A}}\left(L^{\infty}, L^{1}\right)$ be given. Let $Q,\left(p_{n}, q_{n}\right)$, and $G_{n}$ be as given by Proposition 2.1.

Let $\Phi_{1}$ be any continuous, compactly supported function such that $\Phi_{1} \geq$ 0 and $\Phi_{1}(x, y)=1$ for $(x, y) \in \operatorname{supp}(\Phi) Q$. In particular, we have that $\Phi=\Phi \cdot \Phi_{1}$.

We claim that

$$
L_{(x, y)} \Phi \cdot\left(\left(L_{(x, y)} \Phi_{1} \cdot A_{\left(p_{n}, q_{n}\right)} F\right) * G_{n}\right)=L_{(x, y)} \Phi \cdot\left(A_{\left(p_{n}, q_{n}\right)} F * G_{n}\right) .
$$

To see this, suppose that $(a, b) \in \mathbb{A}$ is such that

$$
L_{(x, y)} \Phi(a, b) \neq 0 \text {. }
$$

This implies that $\Phi\left((x, y)^{-1}(a, b)\right) \neq 0$, so $(x, y)^{-1}(a, b) \in \operatorname{supp}(\Phi)$, and hence

$$
(a, b) \in(x, y) \operatorname{supp}(\Phi) .
$$

We must show that for such an $(a, b)$ we have

$$
\left(\left(L_{(x, y)} \Phi_{1} \cdot A_{\left(p_{n}, q_{n}\right)} F\right) * G_{n}\right)(a, b)=\left(A_{\left(p_{n}, q_{n}\right)} F * G_{n}\right)(a, b),
$$

or, in other words, that

$$
\begin{gathered}
\iint_{\mathbb{A}} L_{(x, y)} \Phi_{1}(p, q) A_{\left(p_{n}, q_{n}\right)} F(p, q) G_{n}\left((p, q)^{-1}(a, b)\right) \frac{d p}{p} d q \\
=\iint_{\mathbb{A}} A_{\left(p_{n}, q_{n}\right)} F(p, q) G_{n}\left((p, q)^{-1}(a, b)\right) \frac{d p}{p} d q .
\end{gathered}
$$

To do this, it suffices to show that

$$
G_{n}\left((p, q)^{-1}(a, b)\right) \neq 0 \Longrightarrow L_{(x, y)} \Phi_{1}(p, q)=1 .
$$

So, suppose $(p, q)^{-1}(a, b) \in \operatorname{supp}\left(G_{n}\right) \subseteq Q$. Then, since $Q=Q^{-1}$, we have $(a, b)^{-1}(p, q) \in Q$, and hence

$$
(p, q) \in(a, b) Q \subseteq(x, y) \operatorname{supp}(\Phi) Q .
$$

Consequently, $(x, y)^{-1}(p, q) \in \operatorname{supp}(\Phi) Q$, and therefore by construction of $\Phi_{1}$ we have $\Phi_{1}\left((x, y)^{-1}(p, q)\right)=1$, whence $L_{(x, y)} \Phi_{1}(p, q)=1$. Thus (1) is proved.

Therefore, if we define $K_{n}$ as follows, then we have the following estimates:

$$
\begin{aligned}
K_{n}(x, y) & =\left\|L_{(x, y)} \Phi \cdot\left(F * L_{\left(p_{n}, q_{n}\right)} G_{n}\right)\right\|_{L^{\infty}(\mathbb{A})} \\
& =\left\|L_{(x, y)} \Phi \cdot\left(A_{\left(p_{n}, q_{n}\right)} F * G_{n}\right)\right\|_{L^{\infty}(\mathbb{A})} \quad \text { (by Lemma 2.3) } \\
& =\left\|L_{(x, y)} \Phi \cdot\left(\left(L_{(x, y)} \Phi_{1} \cdot A_{\left(p_{n}, q_{n}\right)} F\right) * G_{n}\right)\right\|_{L^{\infty}(\mathbb{A})}
\end{aligned}
$$


216

$$
\begin{aligned}
& \leq\|\Phi\|_{L^{\infty}(\mathbb{A})}\left\|\left(L_{(x, y)} \Phi_{1} \cdot A_{\left(p_{n}, q_{n}\right)} F\right) * G_{n}\right\|_{L^{\infty}(\mathbb{A})} \\
& =\|\Phi\|_{L^{\infty}(\mathbb{A})} \operatorname{ess~sup~}_{(a, b) \in \mathbb{A}} \mid \iint_{\mathbb{A}} L_{(x, y)} \Phi_{1}(u, v) A_{\left(p_{n}, q_{n}\right)} F(u, v) \\
& \times G_{n}\left((u, v)^{-1}(a, b)\right) \frac{d u}{u} d v \mid \\
& \leq\|\Phi\|_{L^{\infty}(\mathbb{A})}\left\|G_{n}\right\|_{L^{\infty}(\mathbb{A})} \quad \iiint_{\mathbb{A}}\left|A_{\left(p_{n}, q_{n}\right)} F(u, v) \Phi_{1}\left((x, y)^{-1}(u, v)\right)\right| \frac{d u}{u} d v \\
& \quad\|\Phi\|_{L^{\infty}(\mathbb{A})}\left\|G_{n}\right\|_{L^{\infty}(\mathbb{A})} \\
& \quad \times \iint_{\mathbb{A}}\left|A_{\left(p_{n}, q_{n}\right)} F(u, v) \tilde{\Phi}_{1}\left((u, v)^{-1}(x, y)\right)\right| \frac{d u}{u} d v \\
& =\|\Phi\|_{L^{\infty}(\mathbb{A})}\left\|G_{n}\right\|_{L^{\infty}(\mathbb{A})}\left(\left|A_{\left(p_{n}, q_{n}\right)} F\right| *\left|\tilde{\Phi}_{1}\right|\right)(x, y),
\end{aligned}
$$

where $\tilde{\Phi}_{1}(x, y)=\Phi_{1}\left((x, y)^{-1}\right)$. Since $\Phi_{1}$ is continuous and compactly supported, we have by Lemma $2.1(\mathrm{c})$ that $\tilde{\Phi}_{1} \in L^{1}(\mathbb{A})$. Therefore,

$$
\begin{aligned}
\| F * & L_{\left(p_{n}, q_{n}\right)} G_{n} \|_{W_{\mathbb{A}}\left(L^{\infty}, L^{1}\right)} \\
& =\left\|K_{n}\right\|_{L^{1}(\mathbb{A})} \\
& \leq\|\Phi\|_{L^{\infty}(\mathbb{A})}\left\|G_{n}\right\|_{L^{\infty}(\mathbb{A})}\left\|\left|A_{\left(p_{n}, q_{n}\right)} F\right| *\left|\tilde{\Phi}_{1}\right|\right\|_{L^{1}(\mathbb{A})} \\
& \leq\|\Phi\|_{L^{\infty}(\mathbb{A})}\left\|G_{n}\right\|_{L^{\infty}(\mathbb{A})}\left\|A_{\left(p_{n}, q_{n}\right)} F\right\|_{L^{1}(\mathbb{A})}\left\|\tilde{\Phi}_{1}\right\|_{L^{1}(\mathbb{A})} \\
& =C_{1}\left\|G_{n}\right\|_{L^{\infty}(\mathbb{A})}\|F\|_{L^{1}(\mathbb{A})} \quad \text { (by Lemma 2.1), }
\end{aligned}
$$

with $C_{1}$ independent of $F, G$. Hence,

$$
\begin{aligned}
\|F * G\|_{W_{\mathbb{A}}\left(L^{\infty}, L^{1}\right)} & =\left\|F * \sum_{n \in \mathbb{N}} L_{\left(p_{n}, q_{n}\right)} G_{n}\right\|_{W_{\mathbb{A}}\left(L^{\infty}, L^{1}\right)} \\
& \leq \sum_{n \in \mathbb{N}}\left\|F * L_{\left(p_{n}, q_{n}\right)} G_{n}\right\|_{W_{\mathbb{A}}\left(L^{\infty}, L^{1}\right)} \\
& \leq C_{1}\|F\|_{L^{1}(\mathbb{A})} \sum_{n \in \mathbb{N}}\left\|G_{n}\right\|_{L^{\infty}(\mathbb{A})} \\
& \leq C_{2}\|F\|_{L^{1}(\mathbb{A})}\|G\|_{W_{\mathbb{A}}\left(L^{\infty}, L^{1}\right)}
\end{aligned}
$$

with $C_{2}$ independent of $F, G$. Since $\|\cdot\|_{W_{\mathbb{A}}\left(L^{\infty}, L^{1}\right)}$ and $\|\cdot\| \|_{W_{\mathbb{A}}\left(L^{\infty}, L^{1}\right)}$ are equivalent, this completes the proof. 


\section{Acknowledgments}

We are indebted to Hans Feichtinger for extensive discussions regarding amalgam spaces. A portion of the research for this paper was performed while the second author was visiting the School of Mathematics at the Georgia Institute of Technology. This author thanks this department for its hospitality and support during this visit.

\section{References}

1. H. G. Feichtinger, Banach convolution algebras of Wiener type, in: "Functions, Series, Operators," Proc. Conf. Budapest 38, Colloq. Math. Soc. János Bolyai, 1980, 509-524.

2. H. G. Feichtinger and K. Gröchenig, Banach spaces related to integrable group representations and their atomic decompositions, I, J. Funct. Anal., 86 (1989), 307-340.

3. H. G. Feichtinger and K. Gröchenig, Banach spaces related to integrable group representations and their atomic decompositions, II, Monatsh. Math., 108 (1989), 129-148.

4. C. Heil, An introduction to weighted Wiener amalgams, in: R. Ramakrishnan and S. Thangavelu, eds., Proc. International Conference on Wavelets and their Applications (Chennai, January 2002), Allied Publishers, New Delhi (2003), $183-216$

5. C. Heil and G. Kutyniok, The homogeneous approximation property for wavelet frames, preprint (2006).

6. N. Wiener, On the representation of functions by trigonometric integrals, Math. Z., 24 (1926), 575-616.

7. N. Wiener, Tauberian theorems, Ann. of Math., 33 (1932), 1-100.

8. N. Wiener, "The Fourier Integral and Certain of its Applications," MIT Press, Cambridge, 1933. 